\title{
CRIOPRESERVACIÓN DE SEMEN OVINO EMPLEANDO TRES DILUTORES Y CUATRO COMBINACIONES DE AGENTES CRIOPROTECTORES PERMEANTES Y NO PERMEANTES
}

\author{
Ovine Semen Cryopreservation Using Three Extenders and Four \\ Combinations of Permeant and Non Permeant Agents
}
Rocío Sandoval M. ${ }^{1}$, Alexei Santiani A., ${ }^{1,4}$, Luis Ruiz G. ${ }^{1}$, Víctor Leyva V. ${ }^{1}$, Luis Coronado S. ${ }^{2}$ y Alfredo Delgado C. ${ }^{3}$

\section{Resumen}

\begin{abstract}
El objetivo del estudio fue evaluar el efecto de tres dilutores y cuatro combinaciones de dos agentes crioprotectores permeantes más dos no permeantes sobre la calidad del semen ovino post-descongelamiento. Para esto, en una primera fase se evaluaron entre tres dilutores (A, B y C) y el más adecuado se usó en una segunda fase, donde se evaluaron las siguientes combinaciones de agentes crioprotectores permeantes y no permeantes: 1) Glicerol - Trehalosa, 2) Glicerol - Sacarosa, 3) Etilenglicol - Trehalosa y 4) Etilenglicol - Sacarosa. En el experimento 1, se encontró que el Dilutor A mantenía mejor la motilidad progresiva, viabilidad e integridad acrosomal post-descongelamiento en comparación con los dilutores B y C, por lo que el Dilutor A se empleó en el experimento 2. En este ensayo se encontró que la motilidad progresiva, la viabilidad e integridad acrosomal, la termoresistencia y la integridad de membrana plasmática postdescongelamiento fue mejor en los grupos con glicerol-sacarosa y glicerol-trehalosa en comparación con los grupos con etilenglicol-sacarosa y etilenglicol-trehalosa. Esto demuestra que el glicerol es un mejor crioprotector permeante en comparacion con el etilenglicol; sin embargo, no hubo diferencia en el uso de sacarosa o trehalosa. Se concluye que un dilutor con las características del dilutor A, utilizando glicerol más trehalosa o sacarosa, constituye una buena alternativa para la criopreservación de semen ovino.
\end{abstract}

Palabras clave: criopreservación, ovino, agentes crioprotectores permeantes y no permeantes

\section{Abstract}

The objective of the study was to evaluate the effect of three extenders and four combinations of two permeant and two non permeant cryoprotectant agents on the quality of post thaw ram semen. In Experiment 1, three extender were evaluated (A, B, and $\mathrm{C}$ ) in order to select the best for the next step. In Experiment 2, different combinations of cryoprotectant agents were evaluated as follow: 1) Glycerol-Trehalose, 2) GlycerolSucrose, 3) Ethylene glycol-Trehalose, and 4) Ethylene glycol-Sucrose. In experiment 1,

\footnotetext{
${ }^{1}$ Laboratorio de Reproducción Animal, ${ }^{2}$ Laboratorio de Producción Agropecuaria, ${ }^{3}$ Clínica de Animales Mayores, Facultad de Medicina Veterinaria, Universidad Nacional Mayor de San Marcos, Lima

${ }^{4}$ E-mail: asantiania@unmsm.edu.pe
} 
motility, viability and acrosomal integrity in extender A were higher than in extender B and extender $\mathrm{C}$, and therefore, extender $\mathrm{A}$ was used for experiment 2 . In this assay, motility, viability and acrosomal integrity, thermoresistance, and plasmatic membrane integrity were higher in groups Glycerol-sucrose and Glycerol-trehalose in comparison with groups Ethilene glycol-sucrose and Ethilene glycol-trehalose. However, there were not significative differences between sucrose or trehalose. In conclusion, an extender with characteristics of extender A using glycerol plus trehalose or sucrose constitute a good alternative for cryopreservation of ram semen.

Key words: criopreservation, ram, permeant and non permeant cryoprotector agent

\section{INTRODUCCIÓN}

Los resultados de la inseminación artificial con semen congelado resultan hasta ahora insatisfactorios en ovinos. La fertilidad obtenida con semen congelado es menor a la de semen fresco, debido principalmente a una baja viabilidad post-descongelamiento y a un trastorno subletal en la proporción de espermatozoides sobrevivientes (Watson, 2000). Esto se debe a los daños ocasionados en la membrana del espermatozoide durante el proceso de criopreservación, donde se altera la función metabólica del espermatozoide, reduciendo así el número de células viables y ocasionando una capacitación espermática prematura (Maxwell y Watson, 1996). Consecuentemente, los espermatozoides sólo serían viables un corto periodo de tiempo en el tracto reproductivo de la hembra y, por lo tanto, tendrían una menor oportunidad de poder fecundar los ovocitos (Gillan y Maxwell, 1999).

Los daños producidos en los espermatozoide durante el proceso de criopreservación podrían ser prevenidos parcialmente controlando la velocidad de congelamiento, usando un dilutor adecuado y agregando agentes crioprotectores apropiados. En la criopreservación de semen ovino, se ha venido evaluando diferentes tipos de dilutores y agentes crioprotectores (permeantes y no permeantes) obteniéndose resultados muy variables (Molinia et al., 1994 a,b; Gil et al., 2000; Salamon y Maxwell, 2000).
Los crioprotectores permeantes son sustancias que por su bajo peso molecular pueden atravesar la membrana plasmática, donde tienen la función de evitar la formación intracelular de cristales de hielo, así como evitar la excesiva deshidratación causada por la congelación lenta (Medeiros et al., 2002). Entre los más utilizados está el glicerol y el etilenglicol, siendo el primero el que ha mostrado mejores resultados en estudios de criopreservación de semen ovino en relación a la motilidad progresiva post-descongelamiento (Salamon y Maxwell, 2000). No obstante, el etilenglicol ha mostrado un efecto similar o mejor que el glicerol en otras especies (Mantovani et al., 2002). Por otro lado, los crioprotectores no permeantes son sustancias que por su alto peso molecular resultan útiles cuando se aplican velocidades rápidas de congelación, ya que la acción crioprotectora está asociada con su actividad deshidratante y su interacción específica con la membrana fosfolipídica (Aisen et al., 2000). Éstos suelen usarse en asociación con los agentes crioprotectores permeantes. Los crioprotectores no permeantes más utilizados para la congelación de semen de diferentes especies son la sacarosa, rafinosa, trehalosa y lactosa. La adición de trehalosa y sacarosa al dilutor ha obtenido buenos resultados en la criopreservación de semen ovino (Molinia et al., 1994b; Aisen et al., 2000).

\section{Materiales y Métodos}

El estudio se desarrolló en dos fases. La primera fase permitió identificar y selec- 
cionar entre tres dilutores, el que sería incorporado en los tratamientos de la segunda fase del estudio. En esta útima, se evaluó cuatro combinaciones de dos agentes crioprotectores permeantes y dos no permeantes.

El trabajo se ejecutó en el Laboratorio de Reproducción Animal de la Facultad de Medicina Veterinaria de la Universidad Nacional Mayor de San Marcos (FMVUNMSM). Se utilizaron 2 machos ovinos adultos de la raza Merino y 2 Pelibuey, de 2 a 3 años de edad. Las colecciones de semen se realizaron semanalmente en el establo de la FMV-UNMSM, utilizando una vagina artificial de ovino.

\section{Procedimiento experimental}

\section{Experimento 1: Efecto de tres dilutores}

Se evaluaron tres tratamientos:

? Dilutor A (fracción 1: tris $27.1 \mathrm{~g}$, ácido cítrico $14 \mathrm{~g}$, fructosa $10 \mathrm{~g}$ y yema de huevo $10 \%$, diluidos en agua bidestilada csp. $1 \mathrm{~L}$; y fracción 2: glicerol 6\%, trehalosa $76 \mathrm{~g}$ y EDTA $1.5 \mathrm{~g}$, diluidos en la fracción 1 csp. $1 \mathrm{~L})$.

? Dilutor B (fracción 1: tris 18.17 g, ácido cítrico $9.6 \mathrm{~g}$, fructosa $29.72 \mathrm{~g}$ y yema de huevo $10 \%$, diluidos en agua bidestilada csp. $1 \mathrm{~L}$; y fracción 2: glicerol 6\%, trehalosa $76 \mathrm{~g}$ y EDTA $1.5 \mathrm{~g}$, diluidos en la fracción $1 \mathrm{csp} .1 \mathrm{~L})$.

? Dilutor $\mathrm{C}$ (fracción 1: leche descremada $9.5 \mathrm{~mL}$ y yema de huevo $0.5 \mathrm{~mL}$; fracción 2: fructosa $0.49 \mathrm{~g}$, yema de huevo $0.5 \mathrm{~mL}$, trehalosa $0.89 \mathrm{~g}$, EDTA $0.02 \mathrm{~g} \mathrm{y}$ glicerol $1.47 \mathrm{~mL}$, diluidos en la fracción 1 csp. $10 \mathrm{~mL}$ ).

Se realizaron seis ensayos, empleándose una muestra resultante de cuatro eyaculados en cada ensayo. Se evaluó el efecto de cada dilutor sobre los porcentajes de motilidad progresiva y de espermatozoides vivos con acrosoma intacto post-descongelamiento.
Experimento 2: Efecto de diferentes combinaciones de agentes crioprotectores permeantes y no permeantes

Se empleó como dilutor el que obtuvo los mejores resultados en el Exp. 1. Los tratamientos consistieron en las combinaciones de agentes crioprotectores permeantes y no permeantes: 1) Glicerol-Trehalosa, 2) Glicerol-Sacarosa, 3) Etilenglicol-Trehalosa y 4) Etilenglicol-Sacarosa. Se realizaron ocho ensayos. Se evaluó el efecto de los tratamientos sobre los porcentajes de motilidad progresiva y de espermatozoides vivos con acrosoma intacto post-descongelamiento, termoresis tencia y de espermatozoides con membrana plasmática íntegra.

En cada ensayo, se colectó semen a los cuatro machos. Se trabajó únicamente con las muestras de semen que tuvieron un volumen $>0.8 \mathrm{~mL}$, motilidad masal $>3$ y una concentración $>1000 \times 10^{6}$ de espermatozoides por $\mathrm{mL}$. Los cuatro eyaculados se mezclaron formando un pool de semen.

El enfriamiento del semen comprendió 2 fases: en la primera, se diluyó el semen con la primera fracción del dilutor a $35^{\circ} \mathrm{C}$, luego se disminuyó la temperatura hasta llegar a los $5^{\circ} \mathrm{C}$ en un lapso de 90 minutos. En la segunda fase se agregó la segunda fracción del dilutor (sustancias crioprotectoras), y se incubó a $5{ }^{\circ} \mathrm{C}$ durante 30 minutos para estabilizar la muestra (Salamon y Maxwell, 2000). Luego, las muestras fueron envasadas en pajillas de $0.5 \mathrm{~mL}$. A continuación, las pajillas fueron congeladas con los vapores de nitrógeno líquido; para tal efecto, se disminuyó la temperatura de 5 a $-25^{\circ} \mathrm{C}$ en un lapso de 6 minutos y finalmente fueron sumergidas en nitrógeno líquido $\left(-196{ }^{\circ} \mathrm{C}\right)$ (Byrne et al., 2000).

\section{Mediciones post-descongelamiento}

En el Exp. 1, se evaluó la motilidad progresiva y la viabilidad e integridad acrosomal, mientras que en el Exp. 2, se evaluó; además, la integridad de la membrana plasmática 
y la termoresistencia. El descongelamiento se realizó sumergiendo las pajillas en agua temperada a $37^{\circ} \mathrm{C}$ por 10 segundos. Luego, el semen fue diluido $(1: 10)$ con una solución base isotónica (27.1 g de Tris, 14.0 $\mathrm{g}$ de ácido cítrico, $10.0 \mathrm{~g}$ de fructosa $\mathrm{y}$ agua bidestilada csp. $1,000 \mathrm{~mL}$ ) temperada a $37^{\circ} \mathrm{C}$. Luego de 20 minutos se procedió a la evaluación.

Motilidad progresiva. Se diluyó el semen con suero fisiológico a $37^{\circ} \mathrm{C}$ (1:20). Se colocó una gota sobre una lámina portaobjeto temperada y se cubrió con una lámina cubreobjeto. Se observó en microscopio óptico a 400x, calculándose el porcetanje de espermatozoides con motilidad progresiva.

Viabilidad e integridad acrosomal. Se utilizó la técnica de doble tinción descrita por Didion et al. (1989). Se incubó $50 \mu \mathrm{L}$ de semen con $50 \mu \mathrm{L}$ de Azul Tripan al $2 \%$ por 10 minutos y a $37^{\circ} \mathrm{C}$; luego se realizaron 3 lavados con PBS, centrifugando a $700 \mathrm{~g}$ durante 6 minutos. Se resuspendió el precipitado con PBS y se extendió en un portaobjetos. Este fue sumergido en solución Giemsa al $20 \%$ por 40 minutos. Finalmente, se observó con lente de inmersión (1000x) un mínimo de 200 espermatozoides en diferentes campos ópticos y se calculó el porcentaje de espermatozoides vivos con acrosoma intacto, considerándose como tales aquellos que presentaron un coloración pálida o transparente en la parte posterior a la línea ecuatorial de la cabeza y al mismo tiempo tenían el acrosoma teñido de color fucsia.

Termoresistencia. Se empleó la técnica descrita por Smith y Kaune (2004). Las muestras de semen diluidas en una solución base isotónica fueron incubadas a $37^{\circ} \mathrm{C}$ durante 4 horas, para luego ser sometidas a la técnica de la doble tinción. Se determinó el porcentaje de espermatozoides vivos con acrosoma intacto.

Integridad de membrana plasmática. Los espermatozoides fueron expuestos a un medio hipoosmótico (Jeyendran et al., 1984), compuesto por $0.735 \mathrm{~g}$ de citrato de sodio dihidratado y $1.351 \mathrm{~g}$ de fructosa, disueltos en $100 \mathrm{ml}$ de agua bidestilada. Se mezcló 50 $\mu \mathrm{L}$ de la muestra de semen con $715 \mu \mathrm{L}$ del medio hipoosmótico y se incubó por una hora (Santiani et al., 2004). Esta solución fue extendida sobre un portaobjetos. Se contabilizó un mínimo de 200 espermatozoides y se calculó el porcentaje de espermatozoides con membrana plasmática íntegra, considerándose como tales los que presentaron la cola hinchada.

\section{Análisis Estadístico}

Se empleó el programa estadístico Prism® v. 3.0. Se empleó el análisis de varianza (ANOVA), para determinar diferencias estadísticas entre tratamientos, y la prueba de Tukey para determinar diferencias entre promedios. Previamente, todos los resultados fueron transformados a valores angulares (ángulo $=$ arcoseno $\sqrt{\mathrm{x}}$ ) para acercar los datos a la distribución normal.

Con la finalidad de uniformizar los valores iniciales del semen fresco de cada ensayo, los resultados se expresaron como las proporciones entre los valores inmediatamente descongelados con los valores del semen fresco.

\section{Resultados y Discusión}

\section{Exp. 1: Efecto de tres dilutores}

Los resultados se muestran en el Cuadro 1 . Se puede observar que el dilutor A obtuvo los mayores porcentajes de espermatozoides vivos con acrosoma intacto y de espermatozoides con motilidad progresiva $(\mathrm{p}<0.05)$. Los resultados demuestran que los dilutores que contenían Tris (A y B), tuvieron una mejor capacidad de amortiguación, de regulación osmótica y baja toxicidad en comparación con el dilutor que contenía leche descremada $(\mathrm{C})$. 
Cuadro 1. Valores post-descongelamiento de semen ovino criopreservado con tres dilutores

\begin{tabular}{lcc}
\hline Dilutores & Motilidad Progresiva & $\begin{array}{c}\text { Espermatozoides vivos con } \\
\text { acrosoma intacto }\end{array}$ \\
\hline A & $69.29 \pm 6.76^{\mathrm{a}}$ & $63.12 \pm 4.64^{\mathrm{a}}$ \\
B & $37.40 \pm 5.76^{\mathrm{b}}$ & $34.86 \pm 6.50^{\mathrm{b}}$ \\
C & $23.39 \pm 6.58^{\mathrm{c}}$ & $23.22 \pm 8.23^{\mathrm{c}}$ \\
Semen Fresco & $89.05 \pm 3.76$ & $86.58 \pm 6.08$ \\
\hline${ }^{1}$ Sin diluir $_{\mathrm{a}, \mathrm{b}, \mathrm{c}}$ Letras diferentes dentro de columnas indican diferencias significativas $(\mathrm{p}<0.05)$
\end{tabular}

Las diferencias encontradas entre A y $B$ pueden deberse a las diferencias entre las concentraciones de Tris empleadas por cada dilutor (224 versus $150 \mathrm{mM}$, para A y B, respectivamente). En contraste, Molinia et al. (1994b) encontraron una mejor motilidad progresiva con $150 \mathrm{mM}$ de Tris, cuando compararon diferentes concentraciones (100 a 300 $\mathrm{mM})$. Sin embargo, los resultados encontrados por Molinia et al. (1994a) son inferiores a los reportados por Aisen et al. (2000), quienes utilizaron un dilutor de las mismas características del Dilutor A. Las diferencias en las concentraciones de ácido cítrico y fructosa entre los dilutores A y B, por la necesidad de regular el $\mathrm{pH}$ y la osmoralidad de dichos dilutores, podrían explicar en parte las tendencias observadas entre ambos.

Los resultados obtenidos con el Dilutor $\mathrm{C}$ son diferentes a otros estudios donde no se emplearon crioprotectores no permeantes. Así Gil et al. (2000), al emplear un medio en base a leche descremada, obtuvieron valores de motilidad progresiva superiores en comparación a un dilutor en base a Tris. Este hecho, presumiblemente se debe a que en el presente estudio se empleó un crioprotector no permeante (Trehalosa). Esto corrobora que los altos niveles de calcio presente en la leche pueden afectar negativamente la acción crioprotectora de la Trehalosa (Foote et al., 1993), lo que alteraría la estabilidad de las membranas de los espermatozoides durante el congelamiento.
Exp. 2: Efecto de diferentes combinaciones de agentes crioprotectores permeantes y no permeantes

En este experimento se utilizó el Dilutor A como dilutor base, dado que resultó ser significativamente mejor que los otros dos dilutores. Los resultados se resumen en el Cuadro 2. Se puede apreciar que, independientemente del tipo de crioprotector no permeante empleado, la calidad del semen post-descongelamiento fue significativamente mejor en los grupos que utilizaron glicerol como crioprotector permeante. Por otro lado, no se observó diferencias significativas entre los grupos glicerol-trehalosa versus glicerolsacarosa, ni etileglicol-trehalosa versus etilenglicol-sacarosa.

La ventaja del uso del glicerol sobre el etilenglicol como crioprotector permeante, en la prevención de la pérdida de la motilidad progresiva post-descongelamiento en semen de ovinos ha sido corroborada en otros estudios (Molinia et al., 1994a). Asimismo, en semen caprino se ha encontrado una mejor motilidad progresiva con glicerol en comparación con etilenglicol (Bittencourt et al., 2004). Sin embargo, en protocolos de criopreservación de semen de otras especies, como humanos (Gilmore et al, 2000), equinos (Mantovani et al., 2002) y bovinos (Guthrie et al., 2002), se ha encontrado mejores resultados al usar etilenglicol. 
Cuadro 2. Valores promedio (\%) post-descongelamiento de semen ovino criopreservado con cuatro combinaciones de agentes crioprotectores permeantes y no permeantes

\begin{tabular}{lcccc}
\hline \multicolumn{1}{c}{$\begin{array}{c}\text { Crioprotectores } \\
\text { permeantes + No } \\
\text { permeantes }\end{array}$} & $\begin{array}{c}\text { Motilidad } \\
\text { progresiva }\end{array}$ & $\begin{array}{c}\text { Espermatozoides } \\
\text { vivos con } \\
\text { acrosoma intacto }\end{array}$ & $\begin{array}{c}\text { Termo- } \\
\text { resistencia }\end{array}$ & $\begin{array}{c}\text { Integridad de } \\
\text { membrana }\end{array}$ \\
Glicerol + Trehalosa & $64.1 \pm 4.8^{\mathrm{a}}$ & $57.8 \pm 6.9^{\mathrm{a}}$ & $40.4 \pm 4.4^{\mathrm{a}}$ & $62.7 \pm 2.8^{\mathrm{a}}$ \\
Glicerol + Sacarosa $^{\mathrm{a}}$ & $64.2 \pm 4.4^{\mathrm{a}}$ & $55.7 \pm 6.1^{\mathrm{a}}$ & $37.7 \pm 6.2^{\mathrm{a}}$ & $59.6 \pm 1.3^{\mathrm{a}}$ \\
Etilenglicol + Trehalosa & $44.1 \pm 4.3^{\mathrm{b}}$ & $37.5 \pm 3.9^{\mathrm{b}}$ & $26.7 \pm 6.6^{\mathrm{b}}$ & $40.3 \pm 8.3^{\mathrm{b}}$ \\
Etilenglicol + Sacarosa $^{\mathrm{b}}$ & $48.5 \pm 3.5^{\mathrm{b}}$ & $41.5 \pm 4.1^{\mathrm{b}}$ & $24.9 \pm 3.1^{\mathrm{b}}$ & $37.5 \pm 3.6^{\mathrm{b}}$ \\
Semen Fresco $^{1}$ & $83.6 \pm 7.8$ & $82.5 \pm 2.7$ & & $66.9 \pm 3.6$ \\
\hline
\end{tabular}

${ }^{1}$ No diluido (control)

a,b,c Letras diferentes dentro de columnas indican diferencias significativas $(p<0.05)$

La reducción de la capacidad fecundante del semen criopreservado no sólo se debe a una disminución de la viabilidad espermática, sino además, a los trastornos subletales que experimentan los espermatozoides sobrevivientes (Watson, 2000), de allí la importancia de evaluar la viabilidad e integridad acrosomal, ya que es un indicativo aproximado de la real capacidad fecundante que poseen los espermatozoides postdescongelamiento. En tal sentido, el semen que fue criopreservado con glicerol (más trehalosa o sacarosa), tuvo una mayor proporción de espermatozoides vivos con acrosoma intacto en comparación con el semen criopreservado con etilenglicol (más trehalosa o sacarosa) $(\mathrm{p}<0.05$, Cuadro 2$)$.

De la misma manera, los agentes crioprotectores permeantes empleados en este estudio influyeron significativamente sobre la integridad de membrana plasmática. Se obtuvo un mayor porcentaje de espermatozoides con membrana plasmática íntegra cuando se utilizó glicerol en comparación con etilenglicol. Este parámetro es un indicativo de la funcionabilidad fisiológica de las membranas espermáticas. La alteración en la capacidad de regular el flujo de iones y agua por la membrana plasmática de los espermatozoides, produciría cambios relacionados con la capacitación prematura y la exocitosis acrosomal (Sanocka y Kurpisz, 2004).

Las ventajas de la capacidad crioprotectora del glicerol sobre el etilenglicol en espermatozoides ovinos podría deberse a una mayor susceptibilidad de los espermatozoides ovinos a los efectos tóxicos que podrían tener las altas concentraciones de etilenglicol; por lo tanto, sería conveniente probar el etilenglicol a una menor concentración a la usada en el presente trabajo (2.25\%).

Los crioprotectores no permeantes (trehalosa y sacarosa), no mostraron diferencia significativa en lo concerniente a la motilidad, viabilidad e integridad acrosomal, integridad de membrana y termoresistencia; hecho que coincide con otros reportes, en los cuales se demuestra que protegen a las células espermáticas del efecto de la criopreservación (Aisen et al., 1990). En ese sentido, Aisen et al. (2000) encontraron que la adición de trehalosa en un dilutor en base de Tris mejoró la motilidad progresiva en un $19 \%$. 
La trehalosa es un protector específico de las membranas espermáticas que, como resultado de su interacción con los fosfolípidos, puede unirse con el hidrógeno de las cabezas fosfolipídicas de la membrana, de una manera mas fuerte que el glicerol (Foote et al., 1993). Además, posee una acción crioprotectora relacionada al efecto osmótico, que protege al espermatozoide del congelamiento (Aisen et al., 2002).

Los resultados del presente experimento demuestran que la sacarosa tiene las mismas cualidades de protección que la trehalosa en el espermatozoide ovino durante el proceso de criopreservación. Esto se corrobora con otros estudios, donde no existe diferencia entre el tipo de azúcar (sacarosa, trehalosa) utilizado en el diluyente sobre la motilidad post-descongelamiento de espermatozoides ovinos (Molinia et al., 1994b). Adicionalmente, Aisen et al. (1995) manifiestan que la trehalosa posee una capacidad protectora específica de la membrana que no poseen otros disacáridos como la lactosa.

El presente trabajo es importante para una mejor comprensión del desempeño de los dilutores y agentes crioprotectores en la criopreservación de semen ovino; sin embargo, aún se requieren otros estudios para mejorar la capacidad fecundante del semen criopreservado. Se concluye que un dilutor con las características del dilutor A, utilizando glicerol más trehalosa o sacarosa, constituye una buena alternativa para la criopreservación de semen ovino.

\section{Literatura Citada}

1. Aisen E, Cisale H, Fernandez $H$. 1990. Criopreservación de semen ovino. Nueva técnica. Veterinaria Argentina 7: 176-182.

2. Aisen E, Álvarez H, Venturino A, Larreguy D. 1995. Efecto comparativo de los diluyoconservadores de diferente composición y tonicidad sobre la criopreservación de semen ovino. In- vestigación Agraria: Producción y Sanidad Animal 10: 223-231.

3. Aisen $E$, Álvarez $H$, Venturino A, Garde $J$. 2000. Effect of trehalose and EDTA on cryoprotective action of ram semen diluents. Theriogenology 53: 1053-1061.

4. Aisen E, Medina V, Venturino A. 2002. Cryopreservation and post-thawed fertility of ram semen frozen in different trehalose concentrations. Theriogenology 57: 1801-1808.

5. Bittencourt R, Ribeiro A, Santos A, Fürst $R$. 2004. Freezing goat semen with glycerol and ethylene glycol as the cryoprotective agents. In: $15^{\text {th }}$ International Congress on Animal Reproduction. Brazil.

6. Byrne $G$, Lonergan P, Wade M, Duffy $P$, Donovan A, Hanrahan J, Boland $M .2000$. Effect of freezing rate of ram spermatozoa on subsequent fertility in vivo and in vitro. Anim Reprod Sci 62 : 265-275.

7. Didion B, Dabrinsky J, Giles J, Graves $C$. 1989. Staining procedure to detect viability and the true acrosome reaction in spermatozoa of various species. Gamete Res 22: 51-57.

8. Foote R, Chen Y, Brockett C, Kaproth M. 1993. Fertility of bull spermatozoa frozen in whole milk extender with trehalosa, taurine, or blood serum. J Dairy Sci 76: 1908-1913.

9. Gil J, Söderquist L, RodriguezMartínez H. 2000. Influence of centrifugation and different extenders on post-thaw sperm quality of ram semen. Theriogenology 54: 93-108.

10. Gillan L, Maxwell W. 1999. The functional integrity and fate of cryopreserved ram spermatozoa in the female tract. J Reprod Fertil 54 (Suppl. 1): 271-283.

11. Gilmore, J.; J. Liu; E. Woods; A. Peter; J. Critser. 2000. Cryoprotective agent and temperature effects on human sperm membrane permeabilities: convergence of theoretical and empirical approaches for optimal cryopreservation methods. Hum. Reprod. 15: 335-343. 
12 Guthrie H, Liu, Critser J. 2002. Osmotic tolerant limits and effects of cryoprotectants on motility of bovine spermatozoa. Biol Reprod 67: 1811-1816.

13. Jeyendran R, Van der Ven H, Perez M, Crabo B. 1984. Development of an assay to assess the funtional integrity of the human sperm membrane and its relationship to other semen characteristics. J Reprod Fert. 70: 219-228.

14. Mantovani R, Rora A, Palomo M, Bailoni L, Vicente L. 2002. Comparison between glycerol and ethylene glycol for the cryopreservation of equine spermatozoa. Reprod Nutr Dev 42: 217 226.

15. Maxwell W, Watson P. 1996. Recent progress in the preservation of ram semen. Anim Repro Sci 42: 55-65.

16. Medeiros C, Forell F, Oliveira A, Rodrigues J. 2002. Current status of sperm cryopreservation: why isn't it better?. Theriogenology 57: 327-344.

17. Molinia F, Evans G, Maxwell M. 1994a. Incorporation of penetrating cryoprotectants in diluents for pelletfreezing ram spermatozoa. Theriogenology 42: 849-858.
18. Molinia F, Evans G, Quintana P, Maxwell W. 1994b. Effect of monosaccharides and disaccharides in Trisbased diluent on motility, acrosome integrity and fertility of pellet frozen ram spermatozoa. Anim Repro Sci 36: 113-122.

19. Salamon S, Maxwell W. 2000. Storage of ram semen. Anim.Repro Sci 62: 77111.

20. Sanocka D, Kurpisz M. 2004. Reactive oxygen species and sperm cells. Reprod Biol Endocrinol 2: 12-18.

21. Santiani A, Sandoval R, Ruiz L, Coronado $L$. 2004. Estudio de la integridad en espermatozoides de ovino mediante la prueba de estrés hipoosmótico. En: XXVII Reunión Científica Anual de la APPA. Piura, Perú.

22. Smith R, Kaune H. 2004. Ensayos de función espermática. En: Curso Teórico-Práctico: Entrenamiento en técnicas de análisis seminal. ISA-SAGACH. Temuco, Chile.

23. Watson P. 2000. The causes of reduced fertility with cryopreserved semen. Anim Repro Sci 60-61: 481-492. 